\title{
A MODELING TOOL FOR JOINING RF COMMUNICATIONS WITH IP NETWORKS
}

\author{
David R. Beering \\ Shirley Tseng \\ Jeffrey L. Hayden \\ MorganFranklin \\ Corporation \\ West Chicago, IL
}

\author{
Aaron Corder \\ Missile Defense \\ Agency/GMB/DEP \\ Huntsville, AL
}

\author{
Joe Roman \\ Ryan Frederic \\ Applied Defense Solutions \\ Fulton, MD
}

\author{
Ronald Fish \\ Accenture Defense Group \\ San Diego, CA
}

\begin{abstract}
Current communication network design tools are particularly accessible to specialists in network planning and design; but do not provide an interface that is specifically useful for the communication system planners and engineers. Communication and network system architects, engineers, and integrators need access to simple, intuitive tools for performing system trades and analyses of complex, layered, system of systems architectures, such as the end to end communication systems for the Missile Defense Agency (MDA) Ballistic Missile Defense System (BMDS). A new tool for modeling the communication among the multiple disparate nodes in the BMDS into interconnected Internet Protocol (IP) compliant networks is now in development via a Small Business Innovative Research (SBIR) development contract with the MDA. The capability of the tool, called the Communications System Taxonomy (CommTax) Toolkit, is used in this paper to demonstrate how it will be applied to model the network needed to support a typical BMDS scenario.
\end{abstract}

\section{INTRODUCTION}

Today's system of systems (SoS) is characterized by a myriad of options that must all be considered before committing a proposed communication system design to hardware and eventual operational implementation. This complex trade space presents interesting problems for designers; since end-to-end (E2E) communications systems are often so complex that system designers have difficulty keeping track of the trades, constraints, interface requirements, and interdependencies among components [1]. Components could be commercial off the shelf (COTS) components, legacy government off the shelf (GOTS) components, or those procured in parallel by other agencies. Excellent COTS network design tools, such as QualNet and OPNET, exist for planning and designing networks. These tools, which are centered on utilizing networking technologies, also include radio communication node models, but not to the detail needed to actually plan and design those nodes. These networkmodeling tools generally require well-trained network design specialists to operate, and often require interaction with other specialists, such as communication engineers to get their detailed inputs as piecemeal results obtained with manual hand-tuning. Errors and miss-communication are hard to avoid, and large SoS interactions hard to model, simulate, analyze, and validate. Furthermore, those same network tools are usually not available to the radio communication implementers.

Object oriented and model based development, especially in the software architecture community, are starting to be available in product design environments where designers can 'assemble components' to build models of a system for emulation and test. Similar capabilities are needed by communication system designers to enable radio frequency (RF) communication systems to be 'assembled' into complex E2E system models via simple point and click selections from a defined universe of components.

As model based system development and architecture framework techniques, such as model centric design and Department of Defense (DoD) Architecture Framework (DoDAF) [2], become available and used in the Missile Defense Agency (MDA) and other DoD acquisition programs, a migration path for the current RF design environment is needed to contribute communication domain knowledge to new network centric architecture. The DoDAF architecture description views, the Unified Modeling Language (UML) and the System Modeling Language (SysML)[3] are incorporated into tools used to model general SoS architecture and the systems, processes, hardware, and software within the architecture. Tools are now available that convert UML diagrams directly to software code and enable testing of that code. Equivalent innovations in the communication domain are needed to help the communication system designers to focus their component and system design efforts on meeting the needs of the overall SoS architecture.

As more communication systems are incorporated into network centric architecture, the current patchwork of adhoc communication tools becomes unacceptable and unwieldy for meeting the modeling, analysis, development, and validation needs of network centric communication architecture spanning a multitude of independent communication systems that have to work as a SoS to meet stringent MDA mission requirements. 
This paper discusses CommTax, a new type of communication modeling tool and its application to the MDA BMDS Engagement Sequence Groups (ESGs) and their scenarios. CommTax is presently in the second year (2008-2009) of its phase II development within the context of an MDA SBIR project. MorganFranklin Corporation and its partners, Applied Defense Solutions (ADS) and MAXIM Systems, Inc (now Accenture Defense Group), developed CommTax during the nowcompleted Phase I of the SBIR. CommTax features physical views, logical scenario views, and unified summary views of interdependencies among components, interfaces, and protocols in the information system being studied. The most important elements of the CommTax Toolkit are its ability to present RF and network layer dependencies in one unified model and to maintain detailed, static and dynamic instances of that model in a comprehensive database that can also be transformed and integrated with the databases of other modeling tools. The CommTax model recognizes Internet Protocol (IP) networks, protocols, and technologies by conforming to the universally recognized seven-layer Open Systems Interconnection (OSI) model [4]. The CommTax modeling environment provides the tools to integrate networks with RF communications using simple and visual design aids and a detailed database to model the systems, interfaces, and technologies of IP networks and RF communication nodes.

\section{MDA MISSION PROBLEM}

A BMDS scenario involving two ESGs is shown in Figure 1. In the scenario, a target missile is launched from an Asian location. The ESG for the first line of defense for intercepting the target missile is an Aegis ship at sea, its

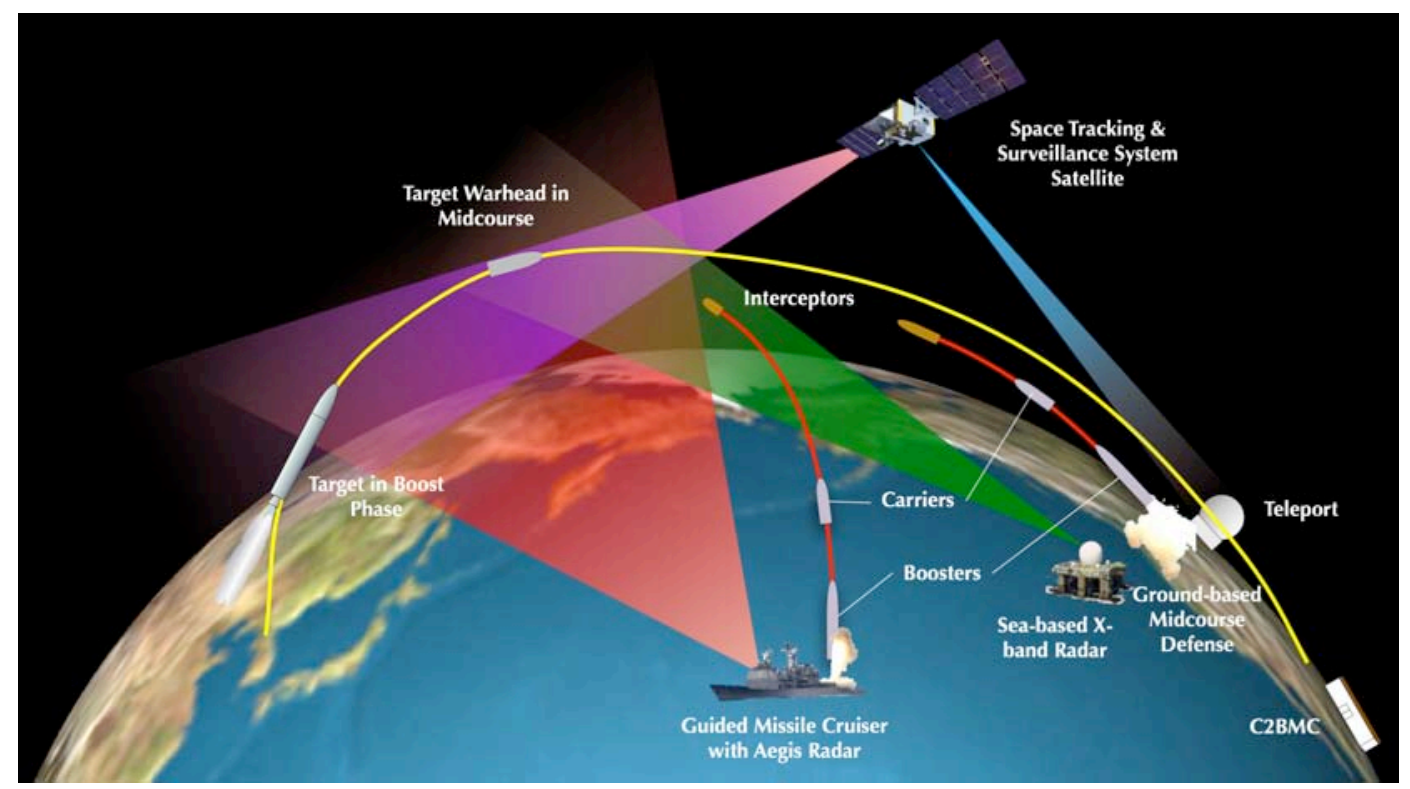

radar, and its on-board interceptor missiles with booster rockets and exoatmospheric kill vehicles (EKVs). This ESG may or may not include satellite communications reachback to fire control command centers in the Continental United States (CONUS). If the target missile in this scenario gets past the first line of defense, a second ESG consisting of a launch detection satellite, a satellite ground terminal, a sea based radar, a ground missile defense (GMD) launch facility, and a command center come into play. We describe below how CommTax would be used to model an ESG scenario similar to one of those in the figure, but first a description of CommTax follows.

\section{COMMTAX TOOLKIT DESCRIPTION}

CommTax integrates and simplifies RF modeling with network modeling to enable RF engineers to collaborate with network engineers on developing the complex communications needed in all MDA areas. CommTax provides a simple graphical user interface (GUI) to manage the complexity of MDA communications systems. Real hardware characteristics are entered into a database via the CommTax GUI. CommTax then uses that information in the communication models that are also entered via the GUI. The database will also be compliant with the DoD Architecture Registry System so that various MDA architecture metadata can be registered with DARS.

The CommTax Toolkit offers system designers, integrators, and operators a common model to describe the system on any scale ranging from subsystems including routers, modems, and amplifiers, to large-scale SoS implementations encompassing regional-, national- or global-scale operations. For the MDA application, CommTax can be applied to missile-to-ground, groundto-missile, and E2E data path designs and descriptions.

The cycle of design, approval, and acceptance is shortened by the simple CommTax GUI, the database, and by providing a common model of the operational picture to all stakeholders during all stages of the system's lifecycle.

The CommTax Toolkit implements OSI standard

Figure 1. BMDS scenario involving two ESGs. 
layers in its RF and network model development. A typical CommTax model consists of nodes connected or arranged in a network. Each node is represented as an object with parameter and variable attributes that describe it. A node is typically capable of transmitting / receiving RF signals and modulating / demodulating digital information to / from the RF respectively. Nodes may also include control centers and other non-RF, network compliant, ground based operation locations. Nodes are connected together in a network either over IP ground lines or through the air by surface and satellite RF links. Communication and network components, such as receivers, transmitters, frequency converters, modulators, coders, encryption devices, routers, etc., are treated within a node as objects that each have their own attributes that may be modeled within CommTax. Actual component attributes and values can be entered into the model as well as idealized values for future components. The fully mature version of the CommTax Toolkit will contain a library of commonly referenced components, such as IP routers, satellite modems, frequency converters, and amplifiers. It will also be possible for the user to import real or idealized components into the database.

CommTax also allows the interfaces between components in the model to be defined. This enables the designer to include the physical attributes of connectors, wiring, and free space characteristics of the wireless links into the network model. Since all of the system's elements are defined by active software models, system designers are able to change key parameters, such as modulation scheme, channel frequency, coding schemes, data rates, interface characteristics, and etc., and see the effects those changes have on other elements in the model and overall system performance. CommTax provides a meaningful visualization of key system attributes.

CommTax models are built in modular blocks to simplify development, to enable integration with existing COTS products, and to provide a solid framework for adding future product features. Microsoft's .NET framework and Visual Studio .NET 2005 make up the development environment for CommTax. The ESRI Company's ArcGIS 9.2 is used for scenario mapping. The Qualnet 4.0 simulation engine [5] is used for discrete element simulation. Database interaction is built using Microsoft SQL Express 2005. Extensible Markup Language (XML) [6] data modeling is done in oXygen and TOPCASED is used for DODAF and UML2 / SysML modeling. TOPCASED and oXygen are both available as plug-ins to the Eclipse open source framework to simplify future integration with agency-specific and corporate-specific development environments and frameworks.
At the presentation layer, CommTax provides user facing GUI modules providing an easy to use design environment for communication designers with simple pick and click parameter values. This simple GUI enables non-RF design professionals, such as system engineers or customers to test models developed in CommTax by varying the parameter values.

CommTax enables communication designers to assemble a complex SoS E2E design via simple GUI point and click selections, activating an underlying component database built from various program component procurement programs, or existing COTS or GOTS components.

At the data access layer, CommTax uses an XML enabled SQL database. Querying for building and extracting the communication and networking component tables and attributes can be performed with SQL queries or XML techniques. The CommTax metadata for the database model is described with a DoDAF Core Architecture Data Model (CADM) compliant XML schema. This will facilitate data transformation for cross-migration of data captured in CommTax communication and networking models with other CADM compliant models developed using other tools for network modeling, architecture definition, dynamic orbit description, software modeling, and hardware design. Portability of CommTax models will be assured by conforming to the CADM metadata XML schema. The CommTax models can then be used to describe and populate CADM system tables, providing data for building DoDAF graphical and tabular system views, particularly the system and services exchange matrix, SV-6. The same XML translation capabilities can be used to import custom model data. The custom model's metadata would be used to identify the parameters and data types and then to translate the model's data into the CommTax database.

The CommTax tool is designed to plug into various network modeling and simulation (NM\&S) tools, including OPNET, although in this release its connectivity is limited to QualNet. The former DoD Network Warfare Simulation (NETWARS) modeling capability has been incorporated into OPNET's Joint Communication Simulation System (JCSS) libraries. Once CommTax supports an OPNET NM\&S engine, the JCSS models can be interchanged with the CommTax environment. CommTax can also model Joint Tactical Radio System (JTRS) nodes by treating each configuration separately as ordinary OSI layer implementations and then by providing switches to handle the JTRS autonomous temporal changes among configurations. In the standalone configuration, CommTax operates in batch mode to calculate model results and doesn't handle mobility. As a 


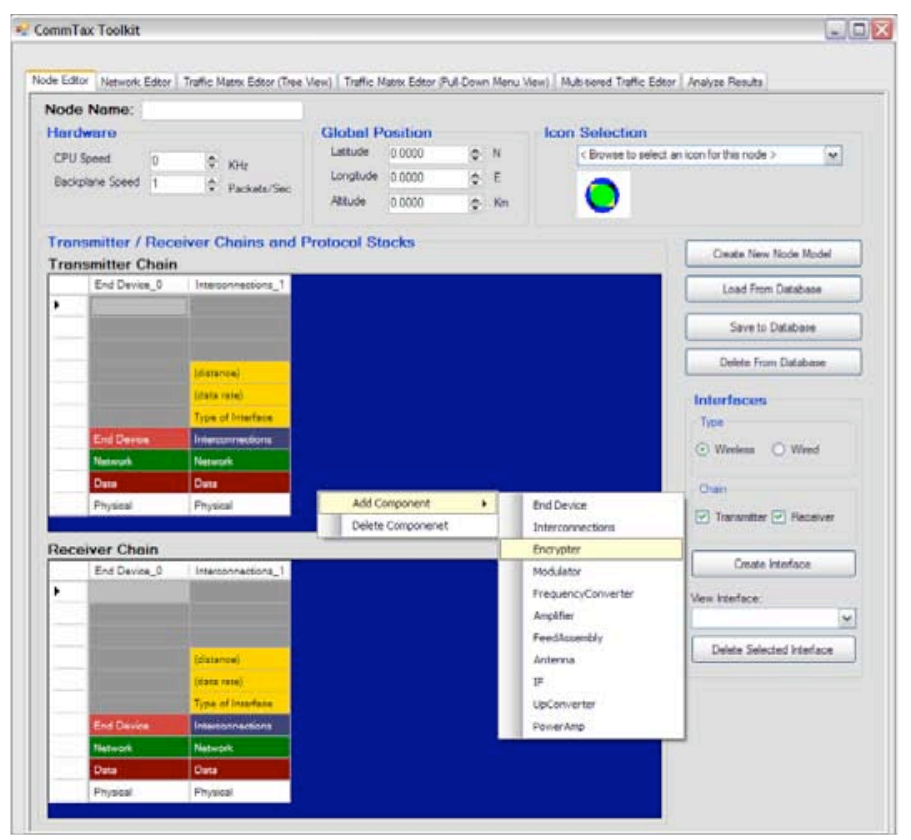

a.

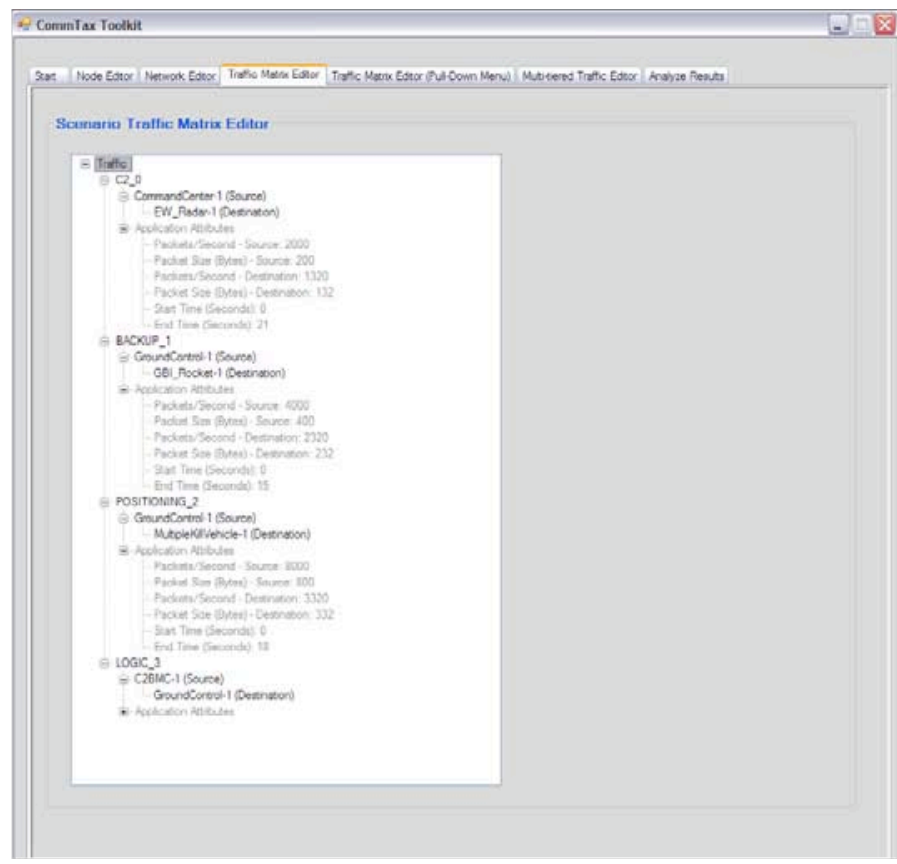

c.

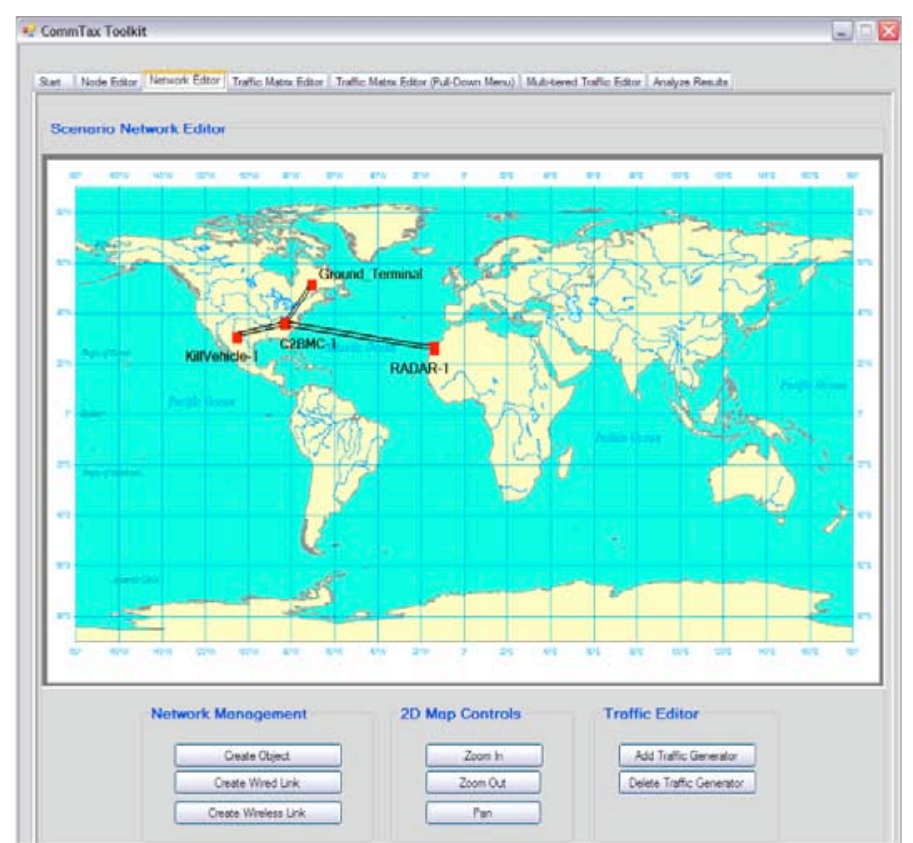

b.

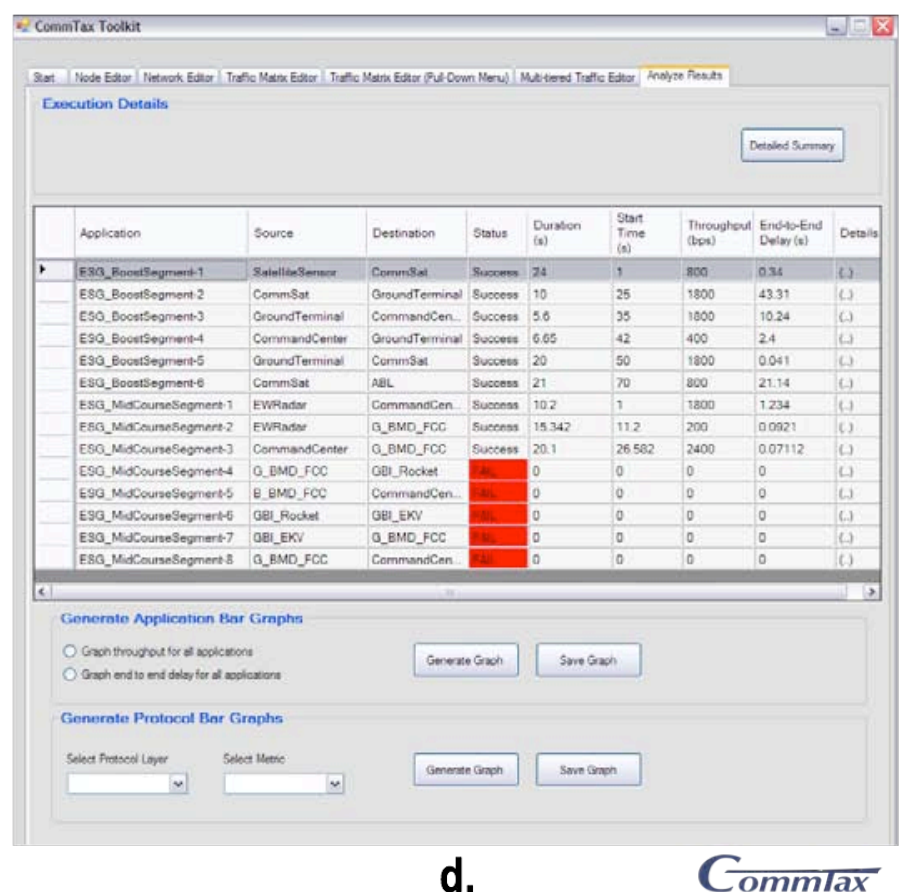

Figure 2. Application GUI windows: a. Node Editor - Communication chain taxonomy and parameters are entered here (shown in editing mode); b. Network Editor - Scenarios are constructed by connecting communication chain nodes in this editor; c. Traffic Editor - Network traffic made of disparate data flows among nodes are constructed in this tree-style editor; and, d. Analysis Screen - Results of a modeled scenario appear here and in printed reports.

plug-in to another NM\&S modeler, CommTax will fully support that modeler's mobility.

Node Editor - This is the first window (Figure 2. a.) seen by the system designer when interacting with the CommTax Toolkit. The Node Editor presents the information system as a series of component blocks and the interconnections between them. This view of the system is similar to a network block diagram. In this window, software models represent all of the components with their key attributes defined. Below each component block is a representation of the ISO seven-layer protocol stack. This gives the system designer an immediate understanding of which layers of the protocol stack each component deals with. 
The connectors between components are also identified in the model. Each connector has attributes that define its characteristics. For instance, a Category 5 Ethernet cable has certain data rates that it can support, along with physical distance limitations depending on the data rates supported. Similarly, an RF channel has many attributes that can become extremely important depending on the frequency of the channel in question. The types of components that can be inserted and configured with CommTax include antennas, cables \& waveguides, power amplifiers, low-noise amplifiers, frequency converters, modulators, encoders, cryptographic modules, satellite modems, and IP routers.

One system that is studied extensively by the Missile Defense Agency is the collection of components that connect interceptors with ground terminals. MDA has conducted extensive testing and analysis on the various conditions that affect connections throughout the flight of an interceptor. Most of the variability in these links comes from the RF channel, which can be affected by a number of impairments. Antennas, frequency converters, and modems are among the components that are critical to successful links. Equally important to these links, however, are other link attributes including modulation scheme, channel coding, and redundancy.

Network Editor - This editor, shown in Figure 2. b., serves as a scenario development window that allows the user to see the system with some of the same attributes that were present in the Node Editor. However, this window presents the system at more of a distance. This view of the system also provides an opportunity for the system designer or evaluator to examine the information loads that are placed on the network. These loads can be bursty or continuous. Loads can also be unidirectional or bi-directional. By understanding the demands that are placed on the network, system designers are better able to determine at what point the network will no longer be able to perform adequately. The example depicted in the figure shows three nodes in CONUS and one in Africa, a radar site, all connected by optical fiber to a central node in Washington, DC.

Traffic Editor - Each data set along the connections in the Network Editor is defined by the data in this window. This window allows the specific attributes of all data flows to be defined, if they are known. For most tactical and strategic defense systems, these flows are well defined and understood, since it is highly important for such a system to exhibit predictable and repeatable performance.

It is often the case that users desire a greater variety of capability than the system designers originally envisioned. By appropriately defining the elements of the Traffic Editor, system designers are able to 'war game' new requirements to determine if the system will support them. System designers can also use this part of the tool to demonstrate the expected efficacy (or failure) of a proposed requirement. An example of a Traffic Editor is shown in Figure 2. c. An example of a scenario that could be considered through this view would be, "what if we wanted to double the data rate of the link?" Alternatively, "what if we wanted to upgrade the video streams from standard definition to high definition?"

Analysis Screen - The final element of the CommTax model is the Analysis Screen shown in Figure 2. d. This view allows the designer to see, at-a-glance, the results of running the model for all the nodes in the entire information system. All of the key attributes of the system are depicted somewhere on this window. The Analysis Screen shown in the figure indicates the data flow breakdown by source-destination connection and by mission phase. Note that the red boxes in the window indicate some of the message-passing requirements were not met in the example test.

CommTax Use Cases - To model and evaluate an end to end system, a user selects or builds the node sets for the mission ESGs in the Node Editor (Figure 2. a); then ties the nodes together into scenarios in the Network Editor (Figure 2. b). For each communication path, receive and transmit node chains (which may be highly asymmetrical and heterogeneous) are visually presented with layers, nodes and parameters, and the user selects, via drop-down boxes, the desired component configurations. Typical application traffic is added in the Traffic Editor (Figure 2. c.) to enable integration with simulation engines, such as QualNet, OPNET, in-house simulation systems, or within CommTax itself in the standalone mode to generate performance result such as data rate, latency, link budget parameters, and target sense to target shoot down time. Atmospheric effect models of weather or nuclear events can be included in the simulation, as needed.

A rich set of reports with views into the analysis database are provided in the Analysis Screen (Figure 2. d.) for reviewing the performance of each configuration by component and for viewing the E2E network system. Once the baseline E2E strings and databases are set, it is easy for system engineers, program managers, or expert implementers to request different alternatives, via dropdown boxes, to converge on an optimal design, perform margin analysis and sensitivity analysis, or investigate piecemeal replacements of alternative segments. As test development components are available or as raw test 
results are available, higher fidelity hardware-in-the-loop (HWIL), mixed simulation can be substituted for simulation-only results. The tool can be used during the entire lifecycle, from design and development in simulation mode, through test, verification, and validation.

Future Directions - Features to consider for incorporation into CommTax, but that are not planned for the current SBIR version, would be to enable CommTax to model communications and networks within an Eclipse framework using SySML and UML2 diagramming and data capture tools such as the open source TOPCASED or any commercial UML2 / SysML tool that could be operated as a plug-in to the open source Eclipse. The concept here would be for CommTax to toggle between the views described in this paper to equivalent UML2 / SySML views at the push of a GUI button.

\section{MODELING A BMDS MISSION}

The BMDS scenario shown in Figure 1 can be modeled at the ESG nodes and connections as shown in Figure 3.

CommTax may be used as follows (the order of actions may vary):

Setup - The following steps are done in the Node Editor: the user populates the major system nodes table in the database with the nodes in the various MDA's ESGs, including: sensors (satellites, radar), ABM systems, command centers, and communication network nodes. The user populates the communication network component table with communication system components used in each major node. Duplicates are consolidated so that a component is entered only once and then subsequently selected from a pick list if used again in other nodes. The OSI layer technology table is populated

\section{Node Editor Screens}

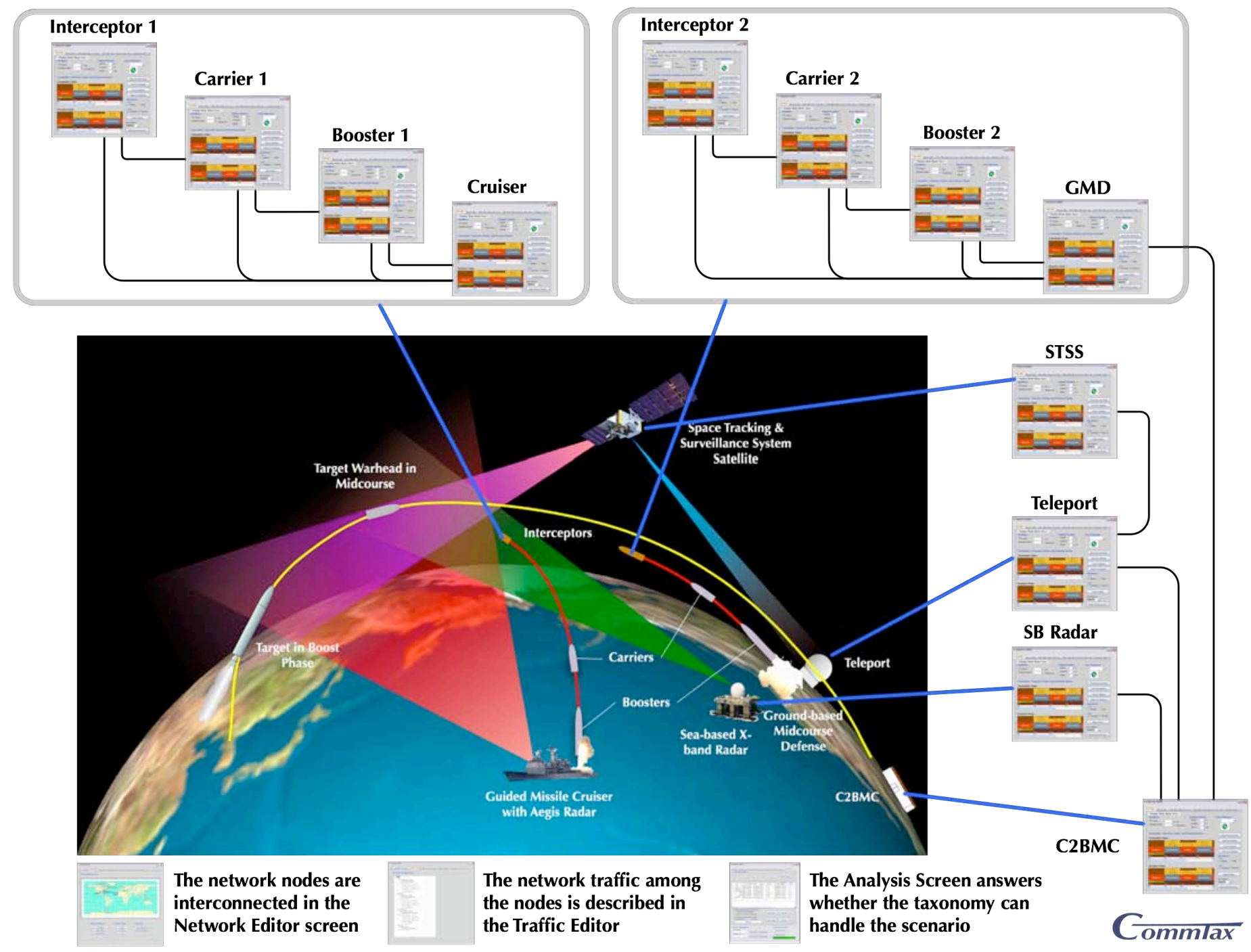

Figure 3. Application of CommTax modeling windows to a BMDS scenario. 
with the types in use. The user then identifies the communication components used by each Sensor, ABM System, Command Center, and Communication Network node.

Select Use Cases - The following steps are done in the Network and Traffic Editors: the user sets up the scenario for one mission segment at a time (target boost, midcourse, or terminal phase). The ESG nodes are selected for that scenario and the user connects the nodes together via their communications systems. The user then populates the Traffic Editor with the data to be passed among the nodes and the characteristics of that data.

Comm Tax Output - The data reported in the Analysis Screen includes: The report of point-to-point data rates and latency and whether the required data rates were passed between the nodes. The target sense to target shoot down time is also reported.

\section{CONCLUSION}

Once the CommTax Toolkit is completed, designers of complex information systems will be able to perform important tradeoff analyses rapidly and accurately using a highly intuitive and visual methodology. Over time a growing library of components, links, node sets, ESG segments and full ESG scenarios will be available to aid the designers. CommTax presents complex systems in a multi-view, intuitive way that will speed time to understanding and will also accelerate development timelines. Moreover, the CommTax Toolkit will give decision makers an important means to interactively and heuristically understand key performance parameters associated with systems that were previously too complex to be understood at all. This has the potential to shorten the time to reach key decisions. Demonstration of the CommTax tool's capabilities is planned for mid-2009.

\section{ACKNOWLEDGMENTS}

This work has been supported by a Missile Defense Agency Small Business Innovation Research contract through Phase I and into Phase II. http://www.dodsbir.net/selections/abs053/mdaabs053.htm

\section{REFERENCES}

[1] Defense Science Board Task Force on Missile Defense Phase III Modeling and Simulation, March 2004, Office of the Under Secretary of Defense For Acquisition, Technology, and Logistics. Washington, D.C. 203013140, available at http://stinet.dtic.mil/oai/oai?verb=getRecord\&metadataPr efix $=$ html\&identifier $=$ ADA423582, accessed on 17 June,
2008.

[2] Department of Defense Architecture Framework (DoDAF) and Core Architecture Data Model (CADM) are available at the Department of Defense Architecture Registry System (DARS), https://dars1.army.mil/IER/index.jsp, accessed on 17 June, 2008.

[3] Unified Modeling Language (UML) and System Modeling Language (SysML) specifications are available at http://www.omg.org/spec/UML/2.1.2/ and http://www.omg.org/spec/SysML/1.0/, accessed on 3 September, 2008.

[4] Open Systems Interconnection (OSI) available at http://www.iso.org/iso/iso_catalogue/catalogue_tc/catalog ue_detail.htm?csnumber $=\overline{2} 0269, \quad$ accessed ${ }^{-}$on 3 September, 2008.

[5] R. Bagrodia, K. Tang, S. Goldman, and D. Kumar, An Accurate, Scalable Communication Effects Server for the FCS System of Systems Simulation Environment, Proceedings of the 2006 Winter Simulation Conference, Monterey, California, USA, December 3-6, 2006

[6] Extensible Markup Language (XML) specifications are available at http://www.w3.org/XML, /XML/Schema, and /Style/XSL accessed on 3 September, 2008. 